\title{
Tumor deposits in rectal adenocarcinoma after neoadjuvant chemoradiation are associated with poor prognosis
}

\author{
Purva Gopal ${ }^{1}$, Pengcheng Lu², Gregory D Ayers ${ }^{2}$, Alan J Herline ${ }^{3}$ and Mary K Washington ${ }^{4}$ \\ ${ }^{1}$ Department of Pathology, University of Texas Southwestern Medical Center, Dallas, TX, USA; ${ }^{2}$ Department \\ of Biostatistics, Division of Cancer Biostatistics, Vanderbilt University Medical Center, Nashville, TN, USA; \\ ${ }^{3}$ Department of Surgery, Vanderbilt University Medical Center, Nashville, TN, USA and ${ }^{4}$ Department of \\ Pathology, Vanderbilt University Medical Center, Nashville, TN, USA
}

\begin{abstract}
Although tumor deposits have been associated with poor prognosis in colorectal carcinoma, the prevalence and clinical significance of tumor deposits in rectal adenocarcinoma following neoadjuvant chemoradiation are relatively unexplored. The aims of this study are to assess the clinical significance of tumor deposits in rectal adenocarcinoma patients, including those receiving neoadjuvant therapy. Pathology slides and medical records from 205 consecutive patients who underwent resection for rectal adenocarcinoma between 1990 and 2010 at a single tertiary care center were reviewed. Patients with tumor deposits had higher tumor grade $(P=\mathbf{0 . 0 0 6})$ and worse tumor stage $(P<0.001)$ at presentation than patients without tumor deposits. Among 110 patients who underwent neoadjuvant chemoradiation, tumor deposits were associated with higher rates of lymph node involvement $(P=0.035)$ and distant metastases $(P=0.006)$, and decreased survival $(P=0.027)$. These patients had a trend toward lower treatment response scores $(P=0.285)$ and higher local recurrence $(P=0.092)$. Of 52 patients with tumor deposits, those who underwent neoadjuvant chemoradiation had significantly worse pretreatment stage by endoscopic ultrasound $(P<0.001)$ but interestingly had significantly lower rates of lymphovascular invasion on resection $(P<0.001)$ compared with those who had not received neoadjuvant chemoradiation. Despite treatment with neoadjuvant chemoradiation, tumor deposits were present in over one-fifth of rectal adenocarcinoma patients. Overall, the outcome of patients with tumor deposits in treated and untreated patients were similar, however the association of tumor deposits with deeply invasive tumors and less tumor regression when comparing with treated patients without tumor deposits raises the possibility that these tumors could have a more aggressive biology, possibly explaining the association of tumor deposits with higher rates of recurrence and lower survival after neoadjuvant chemoradiation. Overall, tumor deposits appear to be a poor prognostic marker among rectal adenocarcinoma patients following neoadjuvant chemoradiation and may identify a subset of patients who require aggressive adjuvant therapy to prevent recurrence.

Modern Pathology (2014) 27, 1281-1287; doi:10.1038/modpathol.2013.239; published online 17 January 2014
\end{abstract}

Keywords: neoadjuvant treatment; rectal carcinoma; tumor deposits

Tumor deposits in the pericolonic and perirectal adipose tissue of patients with colorectal adenocarcinoma were first described in 1935. It was believed at this time that these deposits of colorectal carcinoma were related to vascular invasion, ${ }^{1}$ and this relationship remains one of the predominant

Correspondence: Dr P Gopal, MD, MS, Department of Pathology, University of Texas Southwestern Medical Center, 5959 Harry Hines Blvd POB1, Suite 310, Dallas, TX 75399, USA.

E-mail: purva.gopal@utsouthwestern.edu

Received 19 August 2013; revised 14 November 2013; accepted 26

November 2013; published online 17 January 2014 theories today. Tumor deposits in colorectal carcinoma were first included in the fifth edition of the Tumor Node Metastasis (TNM)/American Joint Committee on Cancer (AJCC) staging manual in 1997, where the deposits were classified based on size. Deposits of colorectal carcinoma $\leq 3 \mathrm{~mm}$ in size were considered a tumor deposit, whereas a deposit $>3 \mathrm{~mm}$ in size was considered a lymph node metastasis. ${ }^{2}$ In the subsequent sixth edition of the AJCC staging manual, tumor deposits were classified based on the contour of the deposit. A tumor deposit with a smooth contour was considered a lymph node metastasis, whereas a tumor 
deposit with an irregular contour was considered venous invasion. ${ }^{3}$ Currently, the seventh edition of the AJCC staging manual classifies tumor deposits based on the following criteria: the deposit should be in the pericolorectal fat or adjacent mesocolic fat, it should be away from the leading edge of the tumor, there should be no evidence of residual lymph node tissue, and finally the tumor deposit should be within the lymph drainage area of the primary carcinoma. ${ }^{4}$

The significance of pericolonic tumor deposits in colorectal adenocarcinoma has been difficult to assess due to repeated changes in TNM classification over the years; in fact, because of the confusion and subjectivity in assessment of tumor deposits, some recent studies propose tumor deposits in colorectal carcinoma be classified as lymph nodes to decrease the subjectivity in assessment of tumor deposits. ${ }^{5,6}$ Regardless, studies have shown that in general, pericolorectal tumor deposits have been associated with higher stage cancers and poor prognosis, including the development of distant metastases, decreased survival, and increased rates of local recurrence. ${ }^{7}$ In patients with rectal adenocarcinoma, tumor deposits tend to occur more commonly in the lower and posterior aspect of the rectum $^{8,9}$ and local recurrence is higher in patients with tumor deposits as compared with those without tumor deposits. ${ }^{10,11}$ However, these prior studies either excluded patients who had received neoadjuvant chemoradiation, or analyzed them as a group with patients who had not received neoadjuvant chemoradiation. $^{8-11}$ Therefore, the significance of tumor deposits in rectal adenocarcinoma, specifically following neoadjuvant chemoradiation, has not been extensively explored, and remains an important question given that patients who receive neoadjuvant chemoradiation are typically those with more advanced tumors and thus the highest risk group for metastatic disease and local recurrence.

The aims of our study were (1) to compare the incidence of tumor deposits between rectal adenocarcinoma patients treated and not treated with neoadjuvant chemoradiation and (2) to determine whether there is an association between tumor deposits and pretreatment tumor stage, treatment response rates, and overall survival among patients treated with neoadjuvant chemoradiation.

\section{Materials and methods}

\section{Study Population}

We retrospectively reviewed records for consecutive patients diagnosed with rectal adenocarcinoma who underwent surgical resection between March 1990 and November 2010 at Vanderbilt University Medical Center. Patients with rectal adenocarcinoma who did not undergo surgical resection were not included in our study. This study was approved by the Institutional Review Board at Vanderbilt University Medical Center.

\section{Data Collection}

Patient demographics, clinical history, and pathologic data were obtained through review of computerized medical records. Age at diagnosis and gender were collected for all patients. Clinical history of interest included pre-treatment stage by endoscopic ultrasound, delivery of neoadjuvant chemoradiation, development of local recurrence, and date/ cause of death. Patients were categorized as died of disease, died of other causes, alive with evidence of disease, and alive with no evidence of disease. Tumor size, the presence or absence of lymphovascular invasion, perineural invasion, lymph node involvement, and distant metastases were extracted from initial pathologic reports.

Resection specimens for all patients were reviewed by a single pathologist (PG) to determine tumor grade, depth of tumor invasion, and the presence or absence of tumor deposits. Tumor deposits were defined as irregular tumor nodules with infiltrative borders in the perirectal adipose tissue, discontinuous from the primary tumor (at least one centimeter from the advancing edge), and lacking a thick fibrous capsule (Figure 1). Treatment response was graded based on College of American Pathologists (CAP) guidelines: 0 , no viable cancer cells; 1 , moderate response; 2 , minimal response; 3 , poor response. ${ }^{12}$ Tumors were staged using the seventh edition of the AJCC staging system. ${ }^{4}$

\section{Statistical Methods}

Continuous variables were summarized using the median and range and compared among patient

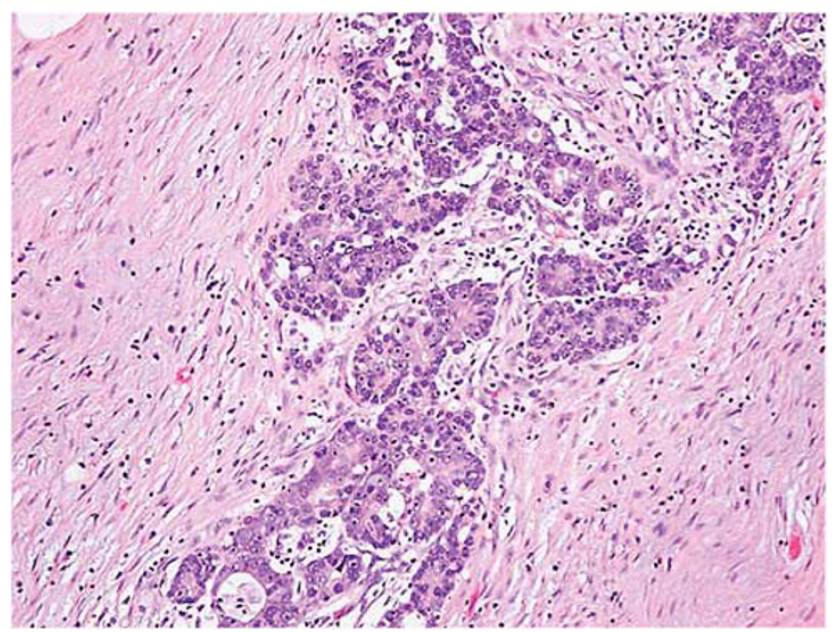

Figure 1 Tumor deposit in rectal adenocarcinoma. Tumor deposits in rectal adenocarcinoma are irregular tumor nodules with infiltrative borders in the perirectal adipose tissue, discontinuous from the primary tumor (at least one centimeter from the advancing edge), and lack a thick fibrous capsule. 
subgroups using the Wilcoxon rank sum test. Categorical variables were summarized as frequencies and percents of total patients in subgroups. Categorical data were compared using the Pearson chi-square test or Fisher's exact test, as appropriate. Ordinary logistic regression or a robust logistic regression method, as warranted, was used to estimate the odds of tumor deposits in the multivariable setting. Overall survival was defined at the time from surgery to death for any reason. Patients alive at the last follow-up were censored. The distributions of overall survival were estimated using the method of Kaplan and Meier and compared among treatment groups using the log-rank test. In supporting analyses, death due to disease and death from other causes were treated as competing risks and the distributions of these two outcomes were estimated using the cumulative incidence function and compared according to Gray for single variables and Fine and Gray in multivariable (proportional hazards) regression. ${ }^{13,14} \mathrm{As}$ with overall survival, patients alive at the time of last follow-up were censored. Cox (proportional hazards) regression was used to assess the impact of tumor deposits on the hazard of overall survival, adjusting for clinically important prognostic variables. Statistical significance is declared for tests with $P$-values $<0.05$. No attempt to control the study-wise type I error rate was made. All analyses were conducted using $\mathrm{R}$ version 2.15.

\section{Results}

\section{Patient Characteristics}

Between March 1990 and November 2010, there were 205 patients with rectal adenocarcinoma who underwent surgical resection (Table 1). The median age of the entire cohort was 59 years old, with $57 \%$ being male. The median follow-up after resection was 14 months (range: 0.25-154 months). Tumor deposits were found on resection in $52(25 \%)$ patients. The median tumor size was $2.5 \mathrm{~cm}$, with significantly larger tumors among those with tumor deposits $(P<0.001)$. Similarly, patients with tumor deposits had higher tumor grade $(P=0.006)$, greater depth of tumor invasion $(P<0.001)$, and higher AJCC stage at presentation $(P<0.001)$ compared with those without tumor deposits. On multivariate logistic regression, the presence of tumor deposits was associated with depth of tumor invasion and AJCC stage. Patients with AJCC stage 3 (OR 12.7, 95\% CI 2.1-78.2) and AJCC stage 4 (OR 19.7, 95\% CI 2.6-147.4) tumors were significantly more likely to have tumor deposits than those with AJCC stage 1 tumors. Associations of tumor grade, tumor size, and neoadjuvant chemoradiation with tumor deposits did not maintain statistical significance in multivariate analysis.
Table 1 Patient characteristics and outcomes by tumor deposit status

\begin{tabular}{|c|c|c|c|}
\hline Variable & $\begin{array}{l}\text { Tumor } \\
\text { deposits, } \\
N=52\end{array}$ & $\begin{array}{l}\text { No tumor } \\
\text { deposits, } \\
N=153\end{array}$ & Test statistic \\
\hline Age (years) & $\begin{array}{c}58.5 \\
(48.5-70.2)\end{array}$ & $\begin{array}{c}59.0 \\
(52.0-68.0)\end{array}$ & $\begin{array}{c}\mathrm{F}_{1}, 203=0.46 \\
P=0.50^{1}\end{array}$ \\
\hline $\begin{array}{l}\text { Gender } \\
\quad \mathrm{F} \\
\mathrm{M}\end{array}$ & $\begin{array}{l}42 \% \\
58 \%\end{array}$ & $\begin{array}{l}43 \% \\
57 \%\end{array}$ & $\begin{array}{l}\chi^{2}{ }_{1}=0.01 \\
P=0.917^{2}\end{array}$ \\
\hline Tumor size (cm) & $\begin{array}{c}3.5 \\
(2.0-4.5)\end{array}$ & $\begin{array}{c}2.0 \\
(0.8-3.5)\end{array}$ & $\begin{array}{c}\mathrm{F}_{1,194}=20.28 \\
\stackrel{P}{<} 0.001^{1}\end{array}$ \\
\hline $\begin{array}{l}\text { Tumor grade } \\
\quad 1 \\
2 \\
3\end{array}$ & $\begin{array}{c}2 \%(1) \\
71 \%(37) \\
27 \%(14)\end{array}$ & $\begin{array}{c}6 \%(9) \\
84 \%(129) \\
10 \%(15)\end{array}$ & 0.006 \\
\hline $\begin{array}{l}\text { Depth of tumor invasion } \\
1 \\
2 \\
3 \\
4\end{array}$ & $\begin{array}{c}0 \%(0) \\
12 \%(6) \\
63 \%(33) \\
25 \%(13)\end{array}$ & $\begin{array}{l}14 \%(22) \\
39 \%(60) \\
39 \%(60) \\
7 \%(11)\end{array}$ & $<0.001$ \\
\hline $\begin{array}{l}\text { AJCC tumor stage } \\
\quad 1 \\
2 \\
3 \\
4\end{array}$ & $\begin{array}{c}2 \%(1) \\
25 \%(13) \\
44 \%(23) \\
29 \%(15)\end{array}$ & $\begin{array}{l}44 \%(67) \\
27 \%(42) \\
22 \%(34) \\
7 \%(10)\end{array}$ & $<0.001$ \\
\hline $\begin{array}{l}\text { Outcome } \\
\text { Died of disease } \\
\text { Died of other causes } \\
\text { Alive }\end{array}$ & $\begin{array}{c}42 \%(22) \\
12 \%(6) \\
46 \%(24)\end{array}$ & $\begin{array}{l}24 \%(37) \\
17 \%(26) \\
59 \%(90)\end{array}$ & 0.043 \\
\hline
\end{tabular}

${ }^{1}$ Wilcoxon test.

${ }^{2}$ Pearson test.

\section{Predictors of Overall Survival}

Median overall survival among all patients was 8.2 years (95\% CI 6.6-13.5 years) (Figure 2). On univariate analysis, the cumulative incidence of disease-specific mortality was significantly higher among patients with tumor deposits compared with those without tumor deposits $(P<0.001)$ (Figure 3$)$ but not by receipt of neoadjuvant chemoradiation $(P=0.957)$. On multivariate analysis, the only variable significantly associated with disease-specific mortality was higher AJCC stage. Patients with AJCC stage 4 tumors had significantly increased hazards (HR 4.17, 95\% CI 1.60-10.84) of mortality compared with those with AJCC stage 1 tumors. On multivariate analysis, tumor deposits were no longer a statistically significant independent predictor of disease-specific mortality (HR 0.81, 95\% CI 0.44-1.46).

\section{Subgroup Analysis Of Neoadjuvant-Treated Patients With And Without Tumor Deposits (Neoadjuvant Chemoradiation + /Tumor Deposit + Patients vs Neoadjuvant Chemoradiation +/Tumor Deposit - Patients)}

Of the 205 rectal adenocarcinoma patients, 110 (54\%) were treated with neoadjuvant chemoradiation. Of these 110 patients, 23 (21\%) had tumor deposits. 


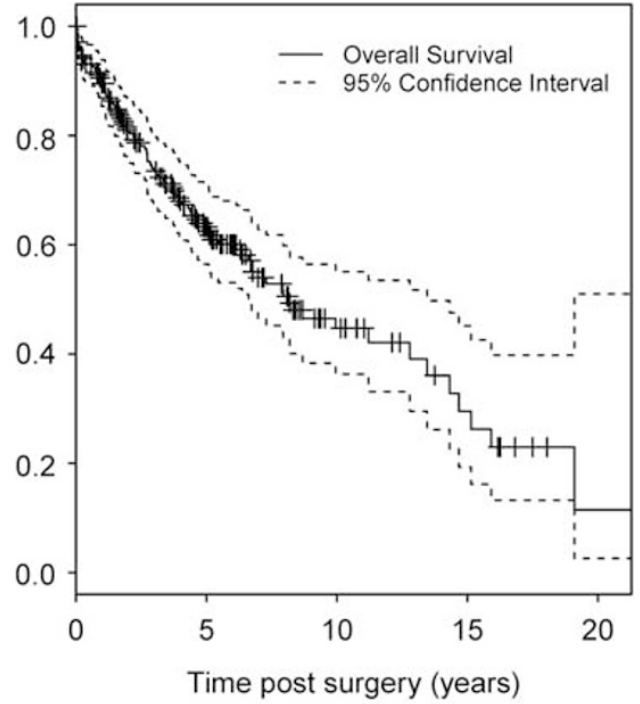

All Patients Number at risk 205
Table 2 Patients with and without tumor deposits following neoadjuvant chemoradiation

\begin{tabular}{|c|c|c|c|}
\hline & $\begin{array}{c}\text { Neoadjuvant } \\
\text { chemoradiation } \\
+/ \text { tumor } \\
\text { deposit }+ \\
\text { patients } \\
(\mathrm{n}=23)\end{array}$ & $\begin{array}{c}\text { Neoadjuvant } \\
\text { chemoradiation } \\
+/ \text { tumor } \\
\text { deposit }- \\
\text { patients } \\
(\mathrm{n}=87)\end{array}$ & $\begin{array}{c}\mathrm{P}- \\
\text { value }\end{array}$ \\
\hline Lymph node Involvement & $13 / 23(57 \%)$ & $28 / 86(33 \%)$ & 0.035 \\
\hline Perineural invasion & $6 / 23(26 \%)$ & $4 / 85(4 \%)$ & 0.002 \\
\hline Lymphovascular invasion & $6 / 22(27 \%)$ & $10 / 85(12 \%)$ & 0.069 \\
\hline Pretreatment stage $\mathrm{T} 3 / \mathrm{T} 4^{\mathrm{a}}$ & $12 / 12(100 \%)$ & $37 / 43(86 \%)$ & 0.170 \\
\hline $\begin{array}{l}\text { Treatment response grade } \\
0-1\end{array}$ & $5 / 23(22 \%)$ & $29 / 87(33 \%)$ & 0.285 \\
\hline $\begin{array}{l}5 \text {-Year local recurrence } \\
\text { rate }\end{array}$ & $19 \%$ & $10 \%$ & 0.092 \\
\hline $\begin{array}{l}5 \text {-Year distant metastases } \\
\text { rate }\end{array}$ & $51 \%$ & $30 \%$ & 0.006 \\
\hline \multicolumn{4}{|l|}{ Overall survival (years) } \\
\hline $\begin{array}{l}\text { Median survival } \\
\text { 1-Year survival }\end{array}$ & $\begin{array}{c}3.1 \\
86 \%\end{array}$ & $\begin{array}{l}11.2 \\
92 \%\end{array}$ & 0.027 \\
\hline
\end{tabular}

a Determined by endoscopic ultrasound.

deposit + patients had a trend toward lower rates of treatment response (grade $0-1)(P=0.285)$ (Table 2).

Five-year local recurrence was 19\% among treated patients with tumor deposits compared with only $10 \%$ among those without tumor deposits $(P=0.092)$. At five years, the incidence of metastatic recurrence among treated tumor deposit-positive patients was $51 \%$ compared with $30 \%$ of treated patients without tumor deposits $(P=0.006)$. The median overall survival among tumor deposit-positive patients was significantly worse than patients without tumor deposits (3.1 vs 11.2 years, $P=0.027$ ). One-year survival rates were 86 and $92 \%$ for tumor deposit and non-tumor deposit patients, respectively.

\section{Subgroup Analysis of Patients with Tumor Deposits at Resection (Neoadjuvant Chemoradiation + /Tumor Deposit + Patients Compared with Neoadjuvant Chemoradiation-/Tumor Deposit + Patients)}

This subset analysis is among the 52 patients whose tissue samples contained tumor deposits. Patients who were neoadjuvant chemoradiation $+/$ tumor deposit + had significantly worse pretreatment stage by endoscopic ultrasound $(P<0.001)$ but were subsequently found to have significantly lower rates of lymphovascular invasion on resection $(P<0.001)$ (Table 3). There were lower rates of lymph node involvement $(P=0.038)$ but similar rates of perineural invasion $(P=0.264)$ and distant metastases at 2 years $(P=0.940)$.

Two-year local recurrence was 19\% among treated patients with tumor deposits and 16\% among untreated tumor deposit-positive patients $(P=0.617)$. Likewise, the incidence of metastatic recurrence 
Table 3 Patients with tumor deposits with or without neoadjuvant chemoradiation

\begin{tabular}{lccr}
\hline & $\begin{array}{c}\text { Neoadjuvant } \\
\text { chemoradiation } \\
\text { +/tumor } \\
\text { deposit } \\
\text { patients } \\
(\mathrm{n}=23)\end{array}$ & $\begin{array}{c}\text { Neoadjuvant } \\
\text { chadiation } \\
\text { deposit }\end{array}$ & \\
& $\begin{array}{c}\text { patients } \\
(\mathrm{n}=29)\end{array}$ & P-value \\
\hline Lymph node involvement & $13 / 23(57 \%)$ & $24 / 29(83 \%)$ & 0.038 \\
Perineural invasion & $6 / 23(26 \%)$ & $4 / 29(14 \%)$ & 0.264 \\
Lymphovascular invasion & $6 / 23(27 \%)$ & $24 / 29(83 \%)$ & $<0.001$ \\
Pretreatment stage T3/T4 & $12 / 12(100 \%)$ & $1 / 5(20 \%)$ & $<0.001$ \\
2-Year local recurrence rate & $19 \%$ & $16 \%$ & 0.617 \\
2-Year distant metastases & $51 \%$ & $46 \%$ & 0.940 \\
rate & & & \\
Overall survival (years) & & & \\
$\quad$ Median survival & 3.1 & 7.1 & 0.715 \\
1-Year survival & $86 \%$ & & \\
\hline
\end{tabular}

${ }^{\mathrm{a}}$ Determined by endoscopic ultrasound.

was similar among treated and untreated tumor deposit-positive patients (51\% vs $46 \%$, respectively, $P=0.940$ ). Median overall survival among neoadjuvant chemoradiation +/tumor deposit + patients was 3.1 years compared with 4.1 years in those without neoadjuvant chemoradiation $(P=0.715)$. One-year survival rates among the treated and untreated patients were $86 \%$ and $79 \%$, respectively.

\section{Discussion}

There are a number of different theories regarding the source of tumor deposits in colorectal carcinoma, however their origin remains uncertain. Tumor deposits were first described in $1935,{ }^{1}$ and at that time it was concluded that they were caused by vascular tumor dissemination, and subsequent studies have shown an association between vascular invasion of the primary tumor and the presence of TD. ${ }^{8,10,11}$ Studies suggest tumor deposits could be the complete replacement of a lymph node by metastatic tumor. This theory has been supported in studies that have shown a correlation with lymph node metastases with extracapsular growth, ${ }^{11}$ and also increased tumor deposits in patients with lymph node metastases as compared with patients with lymph nodes negative for metastases. ${ }^{11,15}$ Finally, another possibility is that tumor deposits could be 'in-transit metastases' as described in melanoma, where tumor cells spread through lymphatic channels and form tumors before reaching lymph nodes. ${ }^{16,17}$ Other studies have examined the growth pattern of tumor deposits, such as perivascular or endovascular growth, perineural growth, or growth within lymphatic channels, however most studies show a mixture of these growth patterns in majority of the cases. Finally, the possibility of tumor deposits that are a short distance from the primary tumor could be an extension from the primary tumor should be considered. This could be due to the manner in which the tumor was sectioned, or possibly discontinuous response to treatment in rectal adenocarcinoma patients who received neoadjuvant chemotherapy. ${ }^{15,18,19}$

Regardless of their origin, tumor deposits in rectal adenocarcinoma have been associated with poor prognosis. In 2003, Prabhudesai et al evaluated whether tumor deposits should be considered an independent prognostic factor in rectal adenocarcinoma patients. Rectal adenocarcinoma patients were divided into two groups, those with tumor deposits (10 who had received neoadjuvant chemoradiation, and 19 who did not) and those without tumor deposits, and found that patients with tumor deposits had distant metastases discovered significantly earlier than the patients without tumor deposits, and patients with tumor deposits had higher vascular invasion (intramural and extramural), lymph node involvement, and perineural invasion. In their study, although there was no significant difference in overall mortality, they observed an association of tumor deposits with other poor prognostic indicators in patients with rectal adenocarcinoma. However, patients with tumor deposits who had received neoadjuvant chemoradiation and those who did not were placed in the same groups with the variable between control groups only being the presence or absence of tumor deposits. ${ }^{8,20}$

Given that tumor deposits in colorectal adenocarcinoma having an association with poor prognosis had already been established, ${ }^{5}$ the goals of our study were to evaluate the significance of tumor deposits in rectal adenocarcinoma in our patient population, and to determine the significance of tumor deposits specifically in rectal adenocarcinoma patients following neoadjuvant chemoradiation. We confirmed that the presence of tumor deposits in patients with rectal adenocarcinoma is associated with a poor prognosis as tumor deposit patients in our study had larger tumors, higher tumor grade, greater tumor invasion, and higher staging at presentation. When comparing patients with tumor deposits following neoadjuvant chemoradiation to patients who did not have tumor deposits following neoadjuvant treatment, patients with tumor deposits had significantly higher positive lymph nodes, perineural invasion, distant metastases, and lower overall survival, similar to the findings of Prabhudesai and colleagues. ${ }^{8}$

Of note, patients in our study who were treated with neoadjuvant chemoradiation and had tumor deposits had significantly decreased lymphovascular invasion when compared with patients with tumor deposits who were not treated preoperatively. This is likely due to the high sensitivity of lymphovascular invasion to chemoradiation that has been described in previous studies. ${ }^{21}$ In addition, patients who were treated preoperatively and had tumor deposits had a significantly higher preoperative stage by endoscopic ultrasound, 
however this difference between these two groups was likely due to patient selection, in that patients with higher preoperative stage would generally be the candidates for preoperative neoadjuvant chemoradiation.

Interestingly, we found that patients with tumor deposits following neoadjuvant chemoradiation also had a higher preoperative clinical stage by endoscopic ultrasound when compared with patients who were treated with neoadjuvant chemoradiation but did not have tumor deposits. Patients who were treated and had tumor deposits trended toward decreased tumor response to neoadjuvant chemoradiation as compared with patients without tumor deposits who were treated, however this difference was not statistically significant possibly due to the difference in the total number of cases between the two groups. However, the majority of the patients in the group with tumor deposits after neoadjuvant chemoradiation had a tumor response grade of 2 or 3 (minimal or no response), with no patients in that group achieving a tumor response grade of 0 (no viable cancer cells). This finding also supports that presence of tumor deposits following neoadjuvant chemoradiation is associated with poor prognosis given that other studies have shown that tumor response to neoadjuvant chemoradiation is an independent prognostic factor in rectal adenocarcinoma patients ${ }^{22}$ and that increased response to neoadjuvant chemoradiation is associated with improved outcomes. ${ }^{23}$

Although our findings are interesting, we acknowledge that our analysis has limitations. As with any retrospective study, there is the possibility of confounders and issues with missing data (eg selection bias). Furthermore, our sample size for subset analyses was only moderately sized so we may have been underpowered to detect some differences. However, we feel these limitations are outweighed by the strengths of our study including its large overall cohort size and that one pathologist reviewed the tumor slides.

Overall, our findings support that the presence of tumor deposits following neoadjuvant chemoradiation is associated with poor prognostic indicators similar to patients with tumor deposits in colorectal adenocarcinoma patients in general. Our findings that patients with tumor deposits who were treated with neoadjuvant chemoradiation trended toward having a decreased tumor regression grade in response to treatment and had a higher pre-treatment clinical stage by endoscopic ultrasound raises the possibility that patients with tumor deposits following neoadjuvant chemoradiation may have more aggressive tumor biology than treated patients without tumor deposits. Given that some tumor deposits in our treated patients had areas with treatment effect around them could suggest that tumor deposits in some patients following neoadjuvant treatment could represent discontinuous eradication of the original tumor; however, further studies would be required to define the mechanism of development of tumor deposits in patients receiving neoadjuvant treatment and whether it is different from tumor deposit development in patients without neoadjuvant treatment. Previous theories of tumor deposit development such as lymphovascular invasion or complete replacement of lymph nodes could also apply to the development of tumor deposits in the setting of neoadjuvant therapy. Regardless of the origin of tumor deposits, in our study, there was an association of the presence of tumor deposits following neoadjuvant treatment with poor prognostic indicators including positive lymph nodes, perineural invasion, distant metastases, and overall survival.

\section{Disclosure/conflict of interest}

The authors declare no conflict of interest.

\section{References}

1 Gabriel WB, Dukes CE, Bussy HJR. Lymphatic spread in cancer of the rectum. Br J Surg 1935;23:395-413.

2 Colon and Rectum. In: Fleming I, Cooper J, Henson D, Hutter R, Kennedy B, Murphy G, O’Sullivan B, Sobin L, Yarbro J (eds). AJCC Cancer Staging Manual, 5th edn. Lippincott-Raven: Philadelphia, 1997, pp 83-90.

3 Colon and Rectum. In: Greene F, Page D, Fleming I, Fritz A, Balch C, Haller D, Morrow M (eds). AJCC Cancer Staging Manual, 6th edn. Springer-Verlag: New York, 2002, pp 127-138

4 Colon and Rectum. In: Edge S, Byrd D, Compton C, Fritz A, Greene F, Trotti A (eds). AJCC Cancer Staging Manual, 7th edn. Springer: New York, Dordrecht, Heidelberg, London, 2009, pp 143-164.

5 Song YX, Gao P, Wang ZN, et al. Can the tumor deposits be counted as metastatic lymph nodes in the UICC TNM staging system for colorectal cancer? PloS One 2012;7:e34087.

6 Ueno H, Mochizuki H, Shirouzu K, et al. Multicenter study for optimal categorization of extramural tumor deposits for colorectal cancer staging. Ann Surg 2012;255:739-746.

7 Nagtegaal ID, Quirke P. Colorectal tumour deposits in the mesorectum and pericolon; a critical review. Histopathology 2007;51:141-149.

8 Prabhudesai A, Arif S, Finlayson CJ, et al. Impact of microscopic extranodal tumor deposits on the outcome of patients with rectal cancer. Dis Colon Rectum 2003;46:1531-1537.

9 Wang Z, Zhou ZG, Wang C, et al. Microscopic spread of low rectal cancer in regions of mesorectum: pathologic assessment with whole-mount sections. WJG 2004;10: 2949-2953.

10 Ueno H, Mochizuki H. Clinical significance of extrabowel skipped cancer infiltration in rectal cancer. Surg Today 1997;27:617-622.

11 Ueno H, Mochizuki H, Tamakuma S. Prognostic significance of extranodal microscopic foci discontinuous with primary lesion in rectal cancer. Dis Colon Rectum 1998;41:55-61. 
12 Ryan R, Gibbons D, Hyland JM, et al. Pathological response following long-course neoadjuvant chemoradiotherapy for locally advanced rectal cancer. Histopathology 2005;47:141-146.

13 Fine J, Gray R. A proportional hazards model for the subdistribution of a competing risk. J Am Statist Assoc 1999;94:496-509.

14 Gray R. A Class of K-sample tests for comparing the cumulative incidence of a competing risk. Ann Stat 1988;16:1141-1154.

15 Belt EJ, van Stijn MF, Bril H, et al. Lymph node negative colorectal cancers with isolated tumor deposits should be classified and treated as stage III. Ann Surg Oncol 2010;17:3203-3211.

16 Grotz TE, Mansfield AS, Kottschade LA, et al. In-transit melanoma: an individualized approach. Oncology. 2011;25:1340-1348.

17 Puppa G, Ueno H, Kayahara M, et al. Tumor deposits are encountered in advanced colorectal cancer and other adenocarcinomas: an expanded classification with implications for colorectal cancer staging system including a unifying concept of in-transit metastases. Modern Pathol 2009;22: 410-415.
18 Puppa G, Sonzogni A, Colombari R, et al. TNM staging system of colorectal carcinoma: a critical appraisal of challenging issues. Arch Pathol Lab Med 2010;134: 837-852.

19 Song JS, Chang HJ, Kim DY, et al. Is the N1c category of the new American Joint Committee on cancer staging system applicable to patients with rectal cancer who receive preoperative chemoradiotherapy? Cancer 2011;117:3917-3924.

20 Puppa G, Maisonneuve P, Sonzogni A, et al. Pathological assessment of pericolonic tumor deposits in advanced colonic carcinoma: relevance to prognosis and tumor staging. Modern Pathol 2007;20:843-855.

21 Shia J, Guillem JG, Moore HG, et al. Patterns of morphologic alteration in residual rectal carcinoma following preoperative chemoradiation and their association with long-term outcome. Am J Surg Pathol 2004;28:215-223.

22 Topova L, Hellmich G, Puffer E, et al. Prognostic value of tumor response to neoadjuvant therapy in rectal carcinoma. Dis Colon Rectum 2011;54:401-411.

23 Biondo S, Navarro M, Marti-Rague J, et al. Response to neoadjuvant therapy for rectal cancer: influence on long-term results. Colorectal Dis 2005;7:472-479. 